\title{
Comparison of effectiveness and time-efficiency between multimedia and conventional counselling on metered-dose inhaler technique education
}

\author{
Teck Long King ${ }^{1}$, BPharm, Evelyn Kui Yee $\underline{K h O}^{1}$, BPharm, Yiek Hung Tiong ${ }^{1}$, BPharm, Siti Norhajariah Binti Julaihi ${ }^{1}$, BPharm
}

INTRODUCTION This study aimed to evaluate whether multimedia counselling (MC) using a touchscreen computer is as effective and time-efficient as conventional counselling (CC) in promoting correct metered-dose inhaler (MDI) technique, with or without the valved holding chamber (VHC).

METHODS Participants in the MDI-only and MDI-with-VHC groups were randomly assigned to the MC group or CC group. No blinding was imposed. Inhalation technique was assessed using checklists before and after counselling. Time spent on counselling was determined for all participants, while time taken to perfect the technique was determined only for participants who achieved perfect technique within one hour.

RESULTS The CC group had more elderly participants than the MC group, but the difference was not significant. MDI-only and MDI-with-VHC users showed significant improvement in their inhaler technique after multimedia $(44.5 \pm 28.0 \%$ and $44.1 \pm 14.4 \%$, respectively) and conventional counselling $(36.8 \pm 20.5 \%$ and $37.0 \pm 14.6 \%$, respectively). No significant difference in MDI technique enhancement was found between the two groups. Although no significant difference was found between the $\mathrm{MC}$ and $\mathrm{CC}$ groups with regard to the time spent on counselling and the time taken to perfect the technique, the average time spent on counselling was longer for MDI-only users. MDI-only users had 13.5 times the odds of failing to achieve perfect technique compared to MDI-with-VHC users ( $95 \%$ confidence interval 1.50-121.32, $p=0.020$ ). CONCLUSION MC and CC significantly improved MDI technique. Both methods showed comparable short-term effectiveness and time-efficiency in MDI technique education. VHC was beneficial, especially for MDI-users with handlung coordination problems.

Keywords: inhaler technique, metered-dose inhaler, multimedia, pharmacy counselling, valved holding chamber

\section{INTRODUCTION}

Improper use of the metered-dose inhaler (MDI) is commonly seen in clinical practice. ${ }^{(1)} \mathrm{A}$ study reported that about $50 \%$ of MDI users did not properly exhale before inhaling and had unsatisfactory breath-holding (less than $3 \mathrm{~s}$ ) after inhalation; some users even inhaled through the nose during and after actuation. ${ }^{(1)}$ Errors in using inhalers may impede respiratory disease control, resulting in the recurrence and exacerbation of the condition. ${ }^{(2)}$ It is therefore important that inhaler users are effectively educated on proper inhaler techniques.

The use of multimedia in patient education involves the utilisation of sounds, images, animations and films to convey messages effectively to patients. A study on the effect of a computer-based multimedia tutorial found that such tutorials were effective in educating patients on inhaler technique as compared to having no intervention at all. ${ }^{(3)}$ In another study involving 105 long-term (average of 13 years) inhaler users, the group of users that was educated on inhaler techniques using multimedia (video) was found to have greater improvement (44\%) than the group that was educated using leaflets (19\%), especially in terms of breathing coordination and breathing-in time. ${ }^{(4)}$ In addition, van der Palen et al showed that the use of video instruction in educating patients on inhaler techniques outperformed both the control group and those who received personal instruction; in that same study, van der Palen et al also showed that the long-term effectiveness of video instruction for inhaler technique education may be as good as the long-term effectiveness of group instruction. ${ }^{(5)}$

In the present study, we aimed to compare the effectiveness of multimedia counselling (using a touchscreen computer) with that of conventional, face-to-face counselling, when used for teaching MDI-only and MDI with valved holding chamber (MDI-with-VHC) techniques. We also aimed to evaluate the time-efficiency of these two counselling methods, in order to identify the more time-efficient method, without compromising on counselling quality.

\section{METHODS}

The present study was a prospective randomised controlled trial evaluating the effectiveness and time-efficiency of multimedia counselling (MC). The participants (i.e. patients and/or caregivers of patients) recruited for the present study were equally and randomly assigned into either the intervention group or control group. Participants in the intervention group received MC, while those in the control group received conventional counselling (CC). Approval for this study was granted by the Medical Research and Ethics Committee, Ministry of Health, Malaysia (ID: NMRR-1217-10836). 
The sample size needed for the present study was estimated using the Power and Sample Size Calculation software version 3.0 (Vanderbilt University, Nashville, TN, USA), ${ }^{(6)}$ based on a similar study by van der Palen et al. ${ }^{(5)}$ In order for MC to have $80 \%$ power to show $10 \%$ more improvement than CC in the MDI technique score difference (i.e. post-counselling score percentage minus pre-counselling score percentage), with a two-sided type 1 error probability of 0.05 and a standard deviation of $15 \%$, a total of 72 participants (36 participants in each group) was required. ${ }^{(6)}$

Study participants were enrolled over six months, from December 2011 to May 2012. Patients who met the following criteria were recruited for the present study: (a) had asthma or chronic obstructive pulmonary disease (COPD); (b) were prescribed MDI-only or MDI-with-VHC before the study; and (c) scored four or less (out of six points) in the inhaler technique pre-counselling assessment. Patients who met any of the following criteria were excluded from the study: (a) had asthma or COPD, but no prescription for MDI; (b) newly started on MDI at the time of recruitment; (c) scored more than four (out of six points) in the inhaler technique pre-counselling assessment; and (d) had poor visual acuity, hearing problems and speech disability. Once recruited, the patients or the caregivers of patients were briefed on the study and their informed consents were obtained.

As the six pharmacists who were involved in the study (four of whom were authors of this article) had heavy routine pharmacy workloads, only two of the six were scheduled daily for patient recruitment and data collection, each at Sibu Hospital's Inpatient Satellite 2 Pharmacy and Outpatient Pharmacy. The participants were first assigned into either the MDI-only group or the MDI-with-VHC group at the location of their recruitment. Thereafter, the participants were sequentially divided into either the intervention group or the control group (i.e. every alternate participant was placed in the control group). To assist randomisation, counselling group allocation forms were used to register participants in their assigned groups. For example, at the Inpatient Satellite 2 Pharmacy, the first recruited MDI-only inpatient user was assigned to the MC group and registered using the MDI-only allocation form, the second MDI-only inpatient user was assigned to the CC group, and so on. Likewise, the first MDI-with-VHC inpatient user was assigned to the MC group, and the second MDI-with-VHC inpatient user, to the CC group, and so on. MDI-with-VHC inpatient users were registered using the MDI-with-VHC allocation form. The same procedure was followed for MDI-only and MDI-with-VHC outpatient users.

In the present study, assessment of inhaler technique was done using two different assessment checklists - one for MDI-only users and the other for MDI-with-VHC users. Both checklists consisted of a list of correct and incorrect steps. Every step performed correctly was awarded with one point, while every incorrect step was given zero point. The checklists were created using the Inhaler Device Assessment Tool by the Nursing Best Practice Research Unit, University of Ottawa, Canada, as a template. ${ }^{(7)}$ The scoring scheme was developed based on a guideline published by the Pharmaceutical Service Division of the Ministry of Health, Malaysia. ${ }^{(8)}$
Once recruited and assigned to a counselling group, each participant was called into a counselling room, where the counselling session (consisting of a series of assessment, counselling and reassessment) was conducted by the same pharmacist who recruited the participant. If the patients were too young to operate their own inhalers, the counselling session was administered to their caregivers. During pre-counselling assessment, the patients or their caregivers were requested to demonstrate their inhaler techniques, which were then assessed using the aforementioned checklists. All scores were recorded.

All the six pharmacists involved in the present study were trained on how to conduct standardised $\mathrm{MC}$ and $\mathrm{CC}$, prior to the start of the study. They were trained to administer either of the counselling methods. In the MC group, the participants were presented with a touchscreen computer (i.e. an Apple ${ }^{\odot} \mathrm{iPad}$ 2) that was preloaded with video tutorials on both MDI-only and MDIwith-VHC techniques. The videos were produced by the hospital's pharmacists and had been used in-house as MDI technique counselling material in the hospital. The instructions in the video tutorial were delivered via narration and reinforced as on-screen text with multilingual support. Multilingual support was a unique feature of MC used in the present study. Participants were allowed to choose their preferred language from four available languages, namely Bahasa Malaysia, Mandarin, English and Bahasa Iban (a native language in Sarawak), for counselling. After they had chosen their preferred language, a stepwise demonstration on inhaler techniques was played. After each step, participants could decide whether to repeat the step or proceed to the next step. They could repeat any step as many times as they wished. After all the steps were completed, a continuous version of the same demonstration was presented again. In the CC group, the participants received the same key information as that received by the $\mathrm{MC}$ group. However, the information was administered by a pharmacist via verbal instruction and physical demonstration using training kits.

Immediately after counselling, the participants in both the $\mathrm{MC}$ and CC groups were reassessed on their inhaler technique. The checklists used were the same as those used for pre-counselling assessment. The difference between pre- and post-counselling inhaler technique score was calculated. If the participants were not able to attain full scores on the first post-counselling assessment, the wrongly performed steps were corrected and they were re-counselled using the assigned counselling method until all steps were correctly performed. Time count was stopped when perfect inhaler technique (i.e. all steps were correct) was achieved. The times at which the counselling session started and stopped were noted. However, the scores were not recorded during the subsequent reassessments of inhaler technique after re-counselling.

Due to logistic and practical concerns, time count was stopped at a maximum of one hour even if the participant had not achieved perfect inhaler technique by that time. As part of pharmacy ethics and professionalism, participants who did not achieve adequate inhaler techniques continued to receive counselling, without restriction on the type of counselling method 
used, until they could use their inhalers effectively. Accessories such as spacers and VHCs, and even other inhaler types (e.g. dry powder inhalers), were also considered, if necessary.

The inhaler technique assessment score was calculated as a percentage (i.e. the number of correctly performed inhalation steps $\div$ the total steps $\times 100 \%$ ). The percentage of participants who correctly performed each inhalation step was also determined. In order to evaluate the time-efficiency of the counselling methods, we recorded the time spent on each counselling session for all participants in the study (i.e. the intention-to-treat population) who achieved or did not achieve full score within one hour, and calculated the average time spent. We also calculated the average time taken to perfect the inhalation technique; the calculation of this average time only included participants who had achieved perfect technique score within one hour.

Independent continuous variables, such as the assessment scores between the two counselling groups, were tested using independent $t$-test. Within-group changes, such as the difference between pre- and post-counselling assessment scores, were tested using paired $t$-test. Categorical variables (e.g. age group, gender, ethnicity, inhalation device used and inpatient/outpatient status) were tested using chi-square analysis. Logistic regression analysis was used to determine the significant factor(s) associated with failure to achieve perfect inhaler technique within one hour. All statistical analyses were performed using the Statistical Package for the Social Sciences software version 20.0 (SPSS Inc, Chicago, IL, USA).

\section{RESULTS}

A total of 72 participants were included in the present study 35 were placed in the MC group, while 37 were placed in the CC group. The characteristics of the participants in both groups are presented in Table I. The CC group had a greater number of elderly (aged > 50 years) participants than the MC group (21 vs. 13 patients), but this difference was not significant. None of these characteristics were significantly different between the two groups.

For MDI-only inhalation, the percentage of participants who correctly performed each inhalation step increased after counselling. This was observed for all steps in the MDI-only inhalation technique and in both counselling groups (Table II). The step in the MDI-only technique that had the lowest percentage of participants correctly performing the step after counselling was Step 4 (i.e. press canister and inhale), for both the $\mathrm{MC}$ and $\mathrm{CC}$ groups. For MDI-with-VHC inhalation, the percentage of participants who correctly performed each inhalation step also increased for all steps after counselling (Table III). After counselling, $100 \%$ correct technique was achieved for several steps (i.e. steps 2-5 for the MC group, and steps 2, 3 and 5 for the CC group).

In terms of assessment scores, participants showed a significant improvement in their scores (by about 40\%), regardless of the counselling method used. For both MDI-only and MDIwith-VHC users, there was no significant difference between the $\mathrm{MC}$ and $\mathrm{CC}$ groups in terms of improvement in the technique assessment (Table IV).
Table I. Characteristics of the participants $(n=72)$.

\begin{tabular}{|c|c|c|c|c|}
\hline \multirow[t]{2}{*}{ Characteristic } & \multicolumn{3}{|c|}{ No. (\%) } & \multirow[t]{2}{*}{ p-value } \\
\hline & $\begin{array}{c}\text { MC } \\
(n=35)\end{array}$ & $\begin{array}{c}\text { CC } \\
(n=37)\end{array}$ & $\begin{array}{c}\text { Total } \\
(n=72)\end{array}$ & \\
\hline Age (yr) & & & & 0.096 \\
\hline$\leq 50$ & $22(57.9)$ & $16(42.1)$ & $38(100.0)$ & \\
\hline$>50$ & $13(38.2)$ & $21(61.8)$ & $34(100.0)$ & \\
\hline Ethnicity & & & & 0.596 \\
\hline Malay & $9(56.3)$ & $7(43.8)$ & $16(100.0)$ & \\
\hline Chinese & $4(36.4)$ & $7(63.6)$ & $11(100.0)$ & \\
\hline Native Sarawakian & $22(48.9)$ & $23(51.1)$ & $45(100.0)$ & \\
\hline Gender & & & & 0.963 \\
\hline Male & $21(48.8)$ & $22(51.2)$ & $43(100.0)$ & \\
\hline Female & $14(48.3)$ & $15(51.7)$ & $29(100.0)$ & \\
\hline Inhalation device used & & & & 0.995 \\
\hline MDI-only & $18(48.6)$ & $19(51.4)$ & $37(100.0)$ & \\
\hline MDI-with-VHC & $17(48.6)$ & $18(51.4)$ & $35(100.0)$ & \\
\hline Type of patient & & & & 0.951 \\
\hline Inpatient & $22(48.9)$ & $23(51.1)$ & $45(100.0)$ & \\
\hline Outpatient & $13(48.1)$ & $14(51.9)$ & $27(100.0)$ & \\
\hline
\end{tabular}

CC: conventional counselling; MC: multimedia counselling; MDI: metered-dose inhaler; VHC: valved holding chamber

Table II. Percentage of participants who correctly performed the inhalation steps, pre- and post-counselling, for metered-dose inhaleronly inhalation.

\begin{tabular}{lccccc}
\hline $\begin{array}{l}\text { Inhalation } \\
\text { step }\end{array}$ & \multicolumn{2}{c}{$\mathbf{M C}(\mathbf{n}=\mathbf{1 8})$} & & \multicolumn{2}{c}{$\mathbf{C C}(\mathbf{n}=\mathbf{1 9})$} \\
\cline { 2 - 3 } \cline { 5 - 6 } & $\begin{array}{c}\text { Pre- } \\
\text { counselling }\end{array}$ & $\begin{array}{c}\text { Post- } \\
\text { counselling }\end{array}$ & & $\begin{array}{c}\text { Pre- } \\
\text { counselling }\end{array}$ & $\begin{array}{c}\text { Post- } \\
\text { counselling }\end{array}$ \\
\hline Step 1 & 44.4 & 77.8 & & 47.4 & 100.0 \\
Step 2 & 5.6 & 66.7 & & 26.3 & 73.7 \\
Step 3 & 61.1 & 100.0 & & 68.4 & 78.9 \\
Step 4 & 16.7 & 55.6 & & 47.4 & 70.7 \\
Step 5 & 27.8 & 72.2 & & 0 & 78.9 \\
Step 6 & 11.1 & 61.1 & & 21.1 & 73.7 \\
\hline
\end{tabular}

Data is presented as percentage. CC: conventional counselling; MC: multimedia counselling; Step 1: remove cap and shake inhaler; Step 2: exhale completely; Step 3: put mouthpiece into mouth and seal properly; Step 4: press canister and inhale; Step 5: hold breath and remove mouthpiece; Step 6: wait for 30-60 s before next puff.

Table III. Percentage of participants who correctly performed the inhalation steps, pre- and post-counselling, for metered-dose inhalerwith-valved holding chamber inhalation.

\begin{tabular}{lccccc}
\hline \multirow{2}{*}{$\begin{array}{l}\text { Inhalation } \\
\text { step }\end{array}$} & \multicolumn{2}{c}{$\mathbf{M C}(\mathbf{n}=\mathbf{1 7})$} & & \multicolumn{2}{c}{$\mathbf{C C}(\mathbf{n}=\mathbf{1 8})$} \\
\cline { 2 - 3 } \cline { 5 - 6 } & $\begin{array}{c}\text { Pre- } \\
\text { counselling }\end{array}$ & $\begin{array}{c}\text { Post- } \\
\text { counselling }\end{array}$ & & $\begin{array}{c}\text { Pre- } \\
\text { counselling }\end{array}$ & $\begin{array}{c}\text { Post- } \\
\text { counselling }\end{array}$ \\
\hline Step 1 & 0 & 91.4 & & 16.7 & 88.9 \\
Step 2 & 94.1 & 100.0 & & 100.0 & 100.0 \\
Step 3 & 47.1 & 100.0 & & 66.7 & 100.0 \\
Step 4 & 76.5 & 100.0 & & 66.7 & 94.4 \\
Step 5 & 70.6 & 100.0 & & 83.3 & 100.0 \\
Step 6 & 6.0 & 76.5 & & 5.6 & 83.3 \\
\hline
\end{tabular}

Data is presented as percentage. CC: conventional counselling; MC: multimedia counselling; Step 1: remove cap and shake inhaler; Step 2: connect inhaler and chamber; Step 3: place mask over mouth and nose with good seal; Step 4: press canister once; Step 5: breathe in and out 5-10 times; Step 6: wait for 30-60 s before next puff.

Intention-to-treat analysis showed no significant difference in the time spent on counselling between the MC and CC groups within one hour (Table V). Overall, $33.1 \pm 24.3$ min were spent 
Table IV. Pre- and post-counselling inhaler technique assessment scores of the participants $(\mathbf{n}=72)$.

\begin{tabular}{|c|c|c|c|c|}
\hline \multirow[t]{2}{*}{ Variable } & \multicolumn{2}{|c|}{ Assessment score } & \multirow[t]{2}{*}{ p-value } & \multirow[t]{2}{*}{ Difference } \\
\hline & Pre-counselling & Post-counselling & & \\
\hline \multicolumn{5}{|c|}{ MDI-only (n = 37) } \\
\hline $\mathrm{MC}$ & $26.8 \pm 20.7$ & $71.3 \pm 29.6$ & $<0.001$ & $44.5 \pm 28.0$ \\
\hline $\mathrm{CC}$ & $38.6 \pm 20.1$ & $75.4 \pm 23.8$ & $<0.001$ & $36.8 \pm 20.5$ \\
\hline p-value & 0.088 & 0.641 & & 0.350 \\
\hline \multicolumn{5}{|c|}{ MDI-with-VHC $(n=35)$} \\
\hline $\mathrm{MC}$ & $51.0 \pm 12.5$ & $95.1 \pm 7.8$ & $<0.001$ & $44.1 \pm 14.4$ \\
\hline $\mathrm{CC}$ & $57.4 \pm 14.3$ & $94.4 \pm 9.9$ & $<0.001$ & $37.0 \pm 14.6$ \\
\hline $\mathrm{p}$-value & 0.165 & 0.832 & & 0.158 \\
\hline
\end{tabular}

Data is presented as mean \pm standard deviation. CC: conventional counselling; MC: multimedia counselling; MDI: metered-dose inhaler; VHC: valved holding chamber

on counselling for MDI-only users, while $14.3 \pm 10.5$ min were spent on counselling for MDI-with-VHC users. Analysis on the time taken to perfect inhaler technique also showed no significant difference between the two counselling groups (Table VI). Overall, MDI-only users needed $12.6 \pm 6.3 \mathrm{~min}$, while MDI-with-VHC users needed $12.9 \pm 7.0 \mathrm{~min}$ to achieve perfect inhaler technique.

Among the 72 participants in the present study, 17 were not able to achieve perfect inhaler technique within one hour - 16 were MDI-only users and one was an MDI-with-VHC user. Simple logistic regression analysis revealed that older age (i.e. $>50$ years of age), MDI-only users and outpatient counselling were significantly associated with failure to perform perfect inhaler technique (Table VII). However, multiple logistic regression analysis showed that only MDI-only users were significantly associated with failure to achieve perfect inhaler technique within one hour $(p=0.020$, adjusted odds ratio 13.49 , 95\% confidence interval 1.50 - 121.32, Table VII). Other variables, such as ethnicity, gender and counselling methods, were not found to be significant.

\section{DISCUSSION}

In the present study, we found that there was improvement in all MDI inhalation steps after counselling, regardless of the counselling method used. This finding is consistent with that of other studies, in which inhaler techniques improved after a pharmacist-led intervention..$^{(9,10)}$ These findings suggest that the administration of simple instructions on correct inhaler technique can result in significant improvement of the technique. We also found that MC using tutorial videos on an Apple ${ }^{\circ}$ iPad 2 was as effective as conventional face-to-face counselling by a pharmacist. This finding contradicts that of van der Palen et al's study, in which MC (i.e. video instruction) was found to be more effective than personal instruction in improving MDI inhalation technique. ${ }^{(5)}$ The difference could be due to the limited tutorial video watching time in the present study. The participants in van der Palen et al's study were allowed unrestricted viewing of the video at home before follow-up, whereas in the present study, most of the participants watched the tutorial only once before the assessment. Therefore, to further improve MDI users' understanding and acquisition of the correct MDI technique, the tutorial videos could be made available after the counselling sessions - either by preparing digital video discs of the video tutorial for distribution or by uploading the video tutorial to an online video streaming website.
Table V. Average time spent on counselling for all participants (i.e. the intention-to-treat population).

\begin{tabular}{llccc}
\hline Device & \multicolumn{4}{c}{ Time spent $(\mathbf{m i n})^{*}$} \\
\cline { 2 - 5 } & \multicolumn{1}{c}{ MC } & CC & p-value & Total \\
\hline MDI-only & $37.1 \pm 24.2$ & $29.3 \pm 24.4$ & 0.339 & $33.1 \pm 24.3$ \\
MDI-with-VHC & $14.4 \pm 8.2$ & $14.2 \pm 12.6$ & 0.946 & $14.3 \pm 10.5$ \\
\hline
\end{tabular}

Data is presented as mean \pm standard deviation. * Maximum time recorded per session is $60 \mathrm{~min}$. CC: conventional counselling; MC: multimedia counselling; MDI: metered-dose inhaler; VHC: valved holding chamber

Table VI. Average time taken by participants to perfect their inhaler technique.

\begin{tabular}{lcccc}
\hline \multirow{2}{*}{ Device } & \multicolumn{4}{c}{ Time taken to perfect technique $(\mathbf{m i n})^{*}$} \\
\cline { 2 - 5 } & MC & CC & p-value & Total \\
\hline MDI-only & $14.1 \pm 7.5$ & $11.4 \pm 5.3$ & 0.344 & $12.6 \pm 6.3$ \\
MDI-with-VHC & $14.4 \pm 8.2$ & $11.5 \pm 5.5$ & 0.228 & $12.9 \pm 7.0$ \\
\hline
\end{tabular}

Data is presented as mean \pm standard deviation. *Calculation excluded participants who did not achieve perfect inhaler technique within one hour. CC: conventional counselling; MC: multimedia counselling; MDI: metered-dose inhaler; VHC: valved holding chamber

In the present study, both counselling methods were found to be equally time-efficient in terms of the time spent on counselling and the time taken to perfect the technique. However, nearly a quarter of the participants were unable to achieve perfect inhaler technique within an hour. Most of these poor performers were MDI-only users and the most prevalent error after counselling was the failure to inhale appropriately with the device (i.e. step 4). Step 4 in the MDI-only inhalation technique requires good hand-lung coordination. Age, comorbidities, cognitive status, hand strength ${ }^{(11,12)}$ and educational level ${ }^{(13)}$ are some factors that may contribute to a patient's difficulty in performing this MDI technique.

The MDI-with-VHC users in the present study performed better than the MDI-only users, suggesting that it is easier to learn the MDI technique with $\mathrm{VHC}$. The hand-lung coordination problem frequently encountered with the use of $\mathrm{MDI}$ can be overcome when VHC is used as an add-on device; this is because $\mathrm{VHC}$ holds the gaseous medication before inhalation, eliminating the need for immediate inhalation after actuation. ${ }^{(14)}$

In Malaysia, particularly in the state of Sarawak, the language barrier between patients and medical information providers is a factor contributing to the complexity in delivering instructions effectively. This led us to believe that multilingual support could 
Table VII. Results of the univariable and multivariable analyses of the factors suspected to be associated with the failure to achieve perfect inhaler technique within one hour.

\begin{tabular}{|c|c|c|c|c|c|c|}
\hline \multirow[t]{2}{*}{ Characteristic } & \multicolumn{2}{|c|}{ No. (\%) } & \multirow[t]{2}{*}{ p-value* } & \multirow[t]{2}{*}{ OR $(95 \% \mathrm{Cl})$} & \multirow[t]{2}{*}{ p-value ${ }^{+}$} & \multirow{2}{*}{$\begin{array}{l}\text { Adjusted OR } \\
(95 \% \mathrm{CI})\end{array}$} \\
\hline & Success $(n=55)$ & Failure $(n=17)$ & & & & \\
\hline \multicolumn{7}{|l|}{ Age (yr) } \\
\hline$>50(n=34)$ & $20(58.8)$ & $14(41.2)$ & 0.003 & $8.17(2.09-31.90)$ & 0.075 & $3.90(0.87-17.38)$ \\
\hline \multicolumn{7}{|l|}{ Ethnicity } \\
\hline Chinese $(n=11)$ & $9(81.8)$ & $2(18.2)$ & & 1.00 (ref) & & NA \\
\hline Native Sarawakian $(n=45)$ & $35(77.8)$ & $10(22.2)$ & 0.770 & $1.29(0.29-6.94)$ & NA & NA \\
\hline \multicolumn{7}{|l|}{ Gender } \\
\hline Male $(n=43)$ & $34(79.1)$ & $9(20.9)$ & & 1.00 (ref) & & NA \\
\hline Female $(n=29)$ & $21(72.4)$ & $8(27.6)$ & 0.515 & $1.44(0.48-4.31)$ & NA & NA \\
\hline \multicolumn{7}{|l|}{ Inhalation device used } \\
\hline MDI-with-VHC (n = 35) & $34(97.1)$ & $1(2.9)$ & & 1.00 (ref) & & 1.00 (ref) \\
\hline \multicolumn{7}{|l|}{ Type of patient } \\
\hline Inpatient $(n=45)$ & $39(86.7)$ & $6(13.3)$ & & 1.00 (ref) & & 1.00 (ref) \\
\hline Outpatient $(n=27)$ & $16(59.3)$ & $11(40.7)$ & 0.011 & $4.47(1.41-14.14)$ & 0.409 & $1.77(0.46-6.89)$ \\
\hline \multicolumn{7}{|l|}{ Type of counselling received } \\
\hline Conventional $(n=37)$ & $29(78.4)$ & $8(21.6)$ & & 1.00 (ref) & & NA \\
\hline Multimedia $(n=35)$ & $26(74.3)$ & $9(25.7)$ & 0.683 & $1.26(0.42-3.73)$ & NA & NA \\
\hline
\end{tabular}

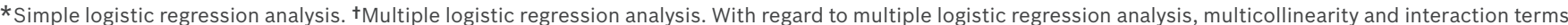
were checked and not found. Hosmer-Lemeshow test $(p=0.885)$, classification table (overall correctly classified percentage $=79.2 \%$ ) and area under the receiving operator characteristic curve (85.0\%) were applied to check model fitness. Cl: confidence interval; MDI: metered-dose inhaler; NA: not applicable; OR: odds ratio; ref: reference group; $\mathrm{VHC}$ : valved holding chamber

potentially minimise or even diminish this barrier. However, we found that MC (which included multilingual support) failed to translate into better inhalation technique when compared to CC. This suggests that neither the language used for the counselling nor the counselling method could determine whether the participants in the present study would achieve perfect technique in the use of the device. Instead, it was the difficulties that the participants faced when using the MDI-only technique that hindered the achievement of a perfect score. Other studies found that these difficulties are associated with a number of factors, including age, hand-lung coordination problems, cultural differences and poor literacy. ${ }^{(11-13,15}$

The present study was not without limitations. First, no followup was done and participants were assessed immediately after counselling. In other words, the results of the present study might only represent the short-term effectiveness of MDI counselling and not its long-term effectiveness. Second, this study was conducted in a single centre and with a small sample size $(n=72)$. Third, no blinding was done. Due to the lack of manpower, the counsellors also served as assessors in the present study. This could have led to bias, as intentional or unintentional teaching, or hint-giving, could have occurred during the assessment. However, it was impossible to blind the participants on the counselling method received, as they were actively involved in the counselling process. Fourth, inter-observer variation could have occurred since multiple assessors were involved. Although checklists were utilised in an attempt to standardise and coordinate the assessment, it was still subject to individual understanding, perception and judgement.

In addition, the CC group was noted to have more elderly participants than the MC group. This disparity was an artefact of the randomisation process, as age was not matched for the two groups. In a study by Allen et al, the authors suggested that the elderly could have difficulty in learning MDI technique due to poor cognitive and executive functions. ${ }^{(12)}$ The uneven distribution of elderly participants in the present study may have resulted in bias in the data obtained on the effect CC had on the MDI-only technique. However, we are unsure of the direction of this bias in the present study. Nevertheless, older age was not found to be significantly associated with failure to achieve perfect technique when it was adjusted for device and inpatient/outpatient status. This could be due to the different backgrounds of the participants in our study population, which comprised mostly native Sarawakians. Other factors, such as low educational level, low socioeconomic status, degree of familiarity with the Apple ${ }^{\circ}$ iPad and preference for the human touch (i.e. CC), were not accounted for. These factors could have affected the participants' ability to achieve perfect MDI technique.

In conclusion, the use of counselling significantly improved $\mathrm{MDI}$ technique (both with and without $\mathrm{VHC}$ ), regardless of the counselling method used. MC was found to be as effective as $\mathrm{CC}$ in teaching correct MDI technique; the time efficiency of both methods was also similar. Therefore, MC could be an effective alternative or a complementary tool in MDI technique counselling. The addition of $\mathrm{VHC}$ is recommended for MDI users, especially if they have hand-lung coordination problems. As the present study evaluated only the short-term effectiveness of counselling, studies on long-term retention of MDI technique after counselling are warranted. To test long-term inhaler technique acquisition, future studies could include follow-up sessions conducted after appropriate time-intervals (e.g. 3-5 months 
following introduction of different counselling methods). ${ }^{(5)}$ Studies can also be conducted to explore the effectiveness of MC versus CC for other types of inhalers, such as Turbuhaler ${ }^{\circledR}$ and Easyhaler ${ }^{\circledR}$.

\section{ACKNOWLEDGEMENTS}

We would like to thank the Director General of Health Malaysia for his permission to publish this paper. This study would not have been completed without the help and cooperation of the staff from Sibu Hospital's pharmacy. We are deeply indebted to Datu Dr Andrew Kiyu Dawie Usop, Dr Flora Ong, Dr Teck-Hock Toh and Dr Marilyn Umar for their valuable comments and reviews. We would also like to express our gratitude to Stella Chuo, Alex Ting and Norazlina binti Othman for their guidance and support; Mr Siew-Tung Wong for proofreading our manuscripts; and Louis Wong and Kai Ning $\mathrm{Ng}$ for their contribution in data collection.

\section{REFERENCES}

1. Melani AS, Bonavia M, Cilenti $\mathrm{V}$, et al. Inhaler mishandling remains common in real life and is associated with reduced disease control. Respir Med 2011; 105:930-8.

2. Hesselink AE, Penninx BW, Wijnhoven HA, Kriegsman DM, van Eijk JT. Determinants of an incorrect inhalation technique in patients with asthma or COPD. Scand J Prim Health Care 2001; 19:255-60.

3. Navarre M, Patel H, Johnson $\mathrm{CE}$, et al. Influence of an interactive computerbased inhaler technique tutorial on patient knowledge and inhaler technique. Ann Pharmacother 2007; 41:216-21.

4. Savage I, Goodyer L. Providing information on metered dose inhaler technique: is multimedia as effective as print? Fam Pract 2003; 20:552-7. 5. van der Palen J, Klein JJ, Kerkhoff AH, van Herwaarden $\mathrm{CL}$, Seydel ER. Evaluation of the long-term effectiveness of three instruction modes for inhaling medicines. Patient Educ Couns 1997; 32(1 Suppl):S87-95.

6. Dupont WD, Plummer WD Jr. Power and sample size calculations. A review and computer program. Control Clin Trials 1990; 11:116-28.

7. Davies B, Danseco E, Cicutto L, et al. Nursing Best Practice Guidelines Evaluation User Guide: Inhaler Device Assessment Tool for Promoting Asthma Control in Children. Canada: Nursing Best Practice Research Unit, University of Ottawa, 2006: 1-30.

8. Handling of Inhaler devices. A Practical Guide for Pharmacist 1st ed. Malaysia: Pharmaceutical Service Division, Ministry of Health, 2010.

9. Hämmerlein A, Müller U, Schulz M. Pharmacist-led intervention study to improve inhalation technique in asthma and COPD patients. J Eval Clin Pract $2011 ; 17: 61-70$

10. Mehuys E, Van Bortel L, De Bolle L, et al. Effectiveness of pharmacist intervention for asthma control improvement. Eur Respir J 2008; 31:790-9.

11. Melani AS, Zanchetta D, Barbato N, et al. Inhalation technique and variables associated with misuse of conventional metered-dose inhalers and newer dry powder inhalers in experienced adults. Ann Allergy Asthma Immunol 2004; 93:439-46.

12. Allen SC, Jain M, Ragab S, Malik N. Acquisition and short-term retention of inhaler techniques require intact executive function in elderly subjects. Age Ageing 2003; 32:299-302.

13. Williams MV, Baker DW, Honig EG, Lee TM, Nowlan A. Inadequate literacy is a barrier to asthma knowledge and self-care. Chest 1998; 114:1008-15.

14. Mitchell JP, Nagel MW. Valved holding chambers (VHCs) for use with pressurised metered-dose inhalers (pMDIs): a review of causes of inconsistent medication delivery. Prim Care Respir J 2007; 16:207-14.

15. Schaafsma ES, Raynor TD, de Jong-van den Berg LT. Accessing medication information by ethnic minorities: barriers and possible solutions. Pharm World Sci 2003; 25:185-90. 